Research Article

\title{
Risk Factors of Tumor Patients with Peripherally Inserted Central Catheter Related Thrombosis and Nursing Intervention Analysis Mediated by Algorithm-Based Ultrasound Images
}

\author{
Lifei Chen $\mathbb{D}^{1}$ and Yingying $\mathrm{Hu} \mathbb{i D}^{2}$ \\ ${ }^{1}$ PICC Clinic, Chenzhou First People's Hospital, Chenzhou 423000, Hunan, China \\ ${ }^{2}$ Department of Oncology, Chenzhou First People's Hospital, Chenzhou 423000, Hunan, China
}

Correspondence should be addressed to Yingying Hu; 170812101166@bitzh.edu.cn

Received 7 June 2021; Revised 30 June 2021; Accepted 12 July 2021; Published 21 July 2021

Academic Editor: Gustavo Ramirez

Copyright ( $\odot 2021$ Lifei Chen and Yingying Hu. This is an open access article distributed under the Creative Commons Attribution License, which permits unrestricted use, distribution, and reproduction in any medium, provided the original work is properly cited.

\begin{abstract}
This paper aimed to explore the risk factors of peripherally inserted central catheter (PICC) related thrombosis (PICC-RT) in tumor patients mediated by ultrasound images under minimum variance (MV) algorithm and put forward the corresponding nursing intervention methods based on the risk factors. The smoothing algorithm, diagonal loading algorithm, and coherence factor algorithm were optimized via MV algorithm. The optimized algorithm was compared with other algorithms to analyze its image quality and image processing speed. Literature retrieval was conducted to analyze the risk factors of PICC-RT in tumor patients. Tumor patients who received PICC for chemotherapy in the hospital from June 2018 to December 2019 were selected. Patients who were accepted before the experiment were taken as the controls, and there were control group (89 cases) and observation group (91 cases). Exercise, average flow rate of axillary vein per unit time, and PICC-RT were compared between the two groups. The results showed that the optimized algorithm (MV-N) had better image resolution, image contrast, and calculation speed than other algorithms. Gender, body mass index (BMI), pathological type, clinical stage, disease history, fibrinogen (FIB), and use of anticoagulant drugs were risk factors for PICC-RT in tumor patients. The number of PICC-RT and complications in the observation group was notably lower in contrast to the control group $(P<0.05)$. It indicated that a novel algorithm was successfully established, which could increase the ultrasonic image quality and computing speed, and upper limb exercise could reduce the incidence of PICC-RT and its complications in tumor patients.
\end{abstract}

\section{Introduction}

PICC is a catheter that is inserted into the central vein through peripheral venipuncture to provide patients with safe and durable venous access [1]. Due to its high safety and convenient operation, it is widely used in intravenous chemotherapy for tumor patients. PICC delivers chemotherapeutic drugs directly to the superior vena cava, reducing the damage to blood vessels caused by irritating drugs [2]. PICC-RT refers to the thrombosis formed on the inner wall of the catheterized vein or the outer wall of the catheter caused by PICC, which is easy to cause pulmonary embolism. About $15 \%$ of PICC-RT patients will develop pulmonary embolism [3]. In recent years, there have been more and more researches on the risk factors of PICC-RT, but the research methods and the time of thrombosis screening are different, and even the final conclusions are inconsistent. Studies have pointed out that upper limb exercise can reduce the incidence of PICC-RT, and inappropriate upper extremity movement may cause other catheterrelated complications [4].

PICC requires to be conducted under ultrasound guidance. Ultrasound imaging technology has the characteristics of easy operation, safety, noninvasiveness, and wide application range. The image quality obtained by commonly used traditional ultrasound system imaging algorithms is poor, which severely restricts the application range of ultrasound imaging technology [5]. In recent years, a variety of 
modern high-definition imaging algorithms have emerged to improve the image quality of ultrasound imaging. However, these algorithms cannot image some detailed scenes when diagnosing some complex diseases. The adaptive beamforming algorithm is based on the MV, which can effectively suppress nonaxial signals, thereby obtaining higher-definition images. Although it can meet the needs of most medical imaging, it is still not possible to provide detailed imaging for some complex diagnoses [6]. The computing graphics processor (General Purpose, GPU) can quickly execute tasks with high computational complexity and has high-performance computing capabilities [7], which provides the possibility for complex imaging applications.

In summary, PICC guided by ultrasound is widely adopted in chemotherapy for cancer patients. However, the speed and image clarity of the current ultrasound algorithm need to be enhanced. Moreover, the results of PICC-RT risk factors are inconsistent, and there is no feasible nursing intervention. Based on GPU, the adaptive beamforming algorithm was optimized. Real-time diagnosis was realized for PICC imaging algorithm of tumor patients, and the risk factors of PICC-RT were analyzed, based on which the feasible nursing intervention measures were put forward.

\section{Materials and Methods}

2.1. Research Subjects and Grouping. Convenience sampling was adopted to collect inpatient tumor patients who underwent chemotherapy with PICC catheter in our hospital from June 2018 to December 2019. Inclusion criteria were I, the tube being placed for the first time, and the coagulation indicators within the normal range before placement and II, patients with Kanowski's health status score more than 60 points. Exclusion criteria were I, patients with high-risk factors such as hypertension, diabetes, and thrombosis history; II, in addition to PICC, the existence of other venous accesses; and III, those who took erythropoiesis drugs before PICC. Patients were grouped according to the order in which patients were included in the study. The 89 cases that met the inclusion criteria from June 2018 to March 2019 were selected as the control group, and the 91 cases that met the inclusion criteria from April 2019 to December 2019 were selected as the observation group. The process had been approved by the ethics committee of the hospital, and all subjects included in the study had signed an informed consent form.

2.2. Adaptive Ultrasound Imaging Algorithm Flow. The adaptive ultrasound imaging algorithm based on the MV algorithm mainly includes three parts: signal delay, weight calculation, and signal superimposition (Figure 1). The signal delay is mainly providing the imaging echo signal data for the corresponding algorithm. The weight calculation part is the core of the entire ultrasound imaging algorithm. The signal-to-noise ratio can be maximized to obtain the apodization weight of the received signal. The signal superposition part mainly refers to the weighted summation of the above apodization weights. Based on the basic content of
$\mathrm{MV}$, the coherence factor and weight calculation method of the signal delay and weight calculation part are optimized. Moreover, the signal in the superimposed part of the signal is adjusted.

2.3. Adaptive Ultrasound Imaging Algorithm. The ultrasound imaging system takes the electrical signal in the probe to emit an ultrasound beam to the imaging target. After receiving the ultrasonic echo, the receiving array element obtains imaging image data through delayed focusing [8]. The algorithm of the one-dimensional probe sensor composed of $N$ elements with equal spacing from the target point to the focused echo signal is as follows:

$$
\bar{E}(t)=E\left(t-\frac{\left\|\overrightarrow{E_{\mathrm{tx}}}-\overrightarrow{E_{p}}\right\|+\left\|\overrightarrow{E_{\mathrm{Ex}}}-\overrightarrow{E_{p}}\right\|}{v}\right) .
$$

In (1), $\overrightarrow{E_{\mathrm{tx}}}, \overrightarrow{E_{\mathrm{Ex}}}$, and $\overrightarrow{E_{p}}$ are the spatial position of the transmitting array element, the receiving array element, and the imaging point, respectively. $t$ is the signal time sequence number; $V$ is the speed of sound; $\bar{E}$ is the echo data, and the algorithm is $\bar{E}=u a+j+m$. $a$ is the steering vector of the delayed echo signal; $j$ and $m$ are the interference signal and noise signal vector, respectively; and $u$ is the desired signal of the target point. The waveform former outputs the spatial measurement data weighting and (2), which is the weight vector of beam forming.

$$
S=\vec{\omega} \bar{E}=\sum_{j=0}^{N-1} \omega_{j} \bar{E}_{j}
$$

The noise covariance matrix estimation algorithm corresponding to the $\mathrm{MV}$ algorithm is $R=1 / N-I+1 \sum_{j=0}^{N-I} x_{j}$, and $x_{j}$ is the $(I \times 1)$ vector. Then the echo signal of the $j$-th group of subapertures is $x_{j}=\left[\bar{E}_{j}, \bar{E}_{j+1}, \ldots \bar{E}_{j+I-1}\right]$, and the adaptive weighting algorithm of the $\mathrm{MV}$ algorithm is as follows:

$$
W=\frac{S^{-1} a}{a S^{-1 \vec{a}}} .
$$

In (3), $\vec{a}$ is the steering vector that has nothing to do with the signal frequency, and the algorithm of the amplitude value of pixel points after the obtained signals are stacked as follows:

$$
S=\frac{1}{N-I+1} \sum_{j=1}^{N-I+1} \vec{\omega} x_{j}
$$

The forward and backward spatial smoothing technique refer to a commonly used noise covariance matrix estimation method in the MV algorithm [9]. The forward spatial smoothing estimation of the conventional MV algorithm is expressed as $R_{f}=R_{m}$, and $R_{m}$ is $\mathrm{MV}$ algorithm spatial smoothing estimation. The backward spatial smoothing estimation is expressed as $R_{b}=J^{2} \cdot R_{f}$, and $J$ is switch matrix. The smoothing estimation algorithm in this article is as follows: 


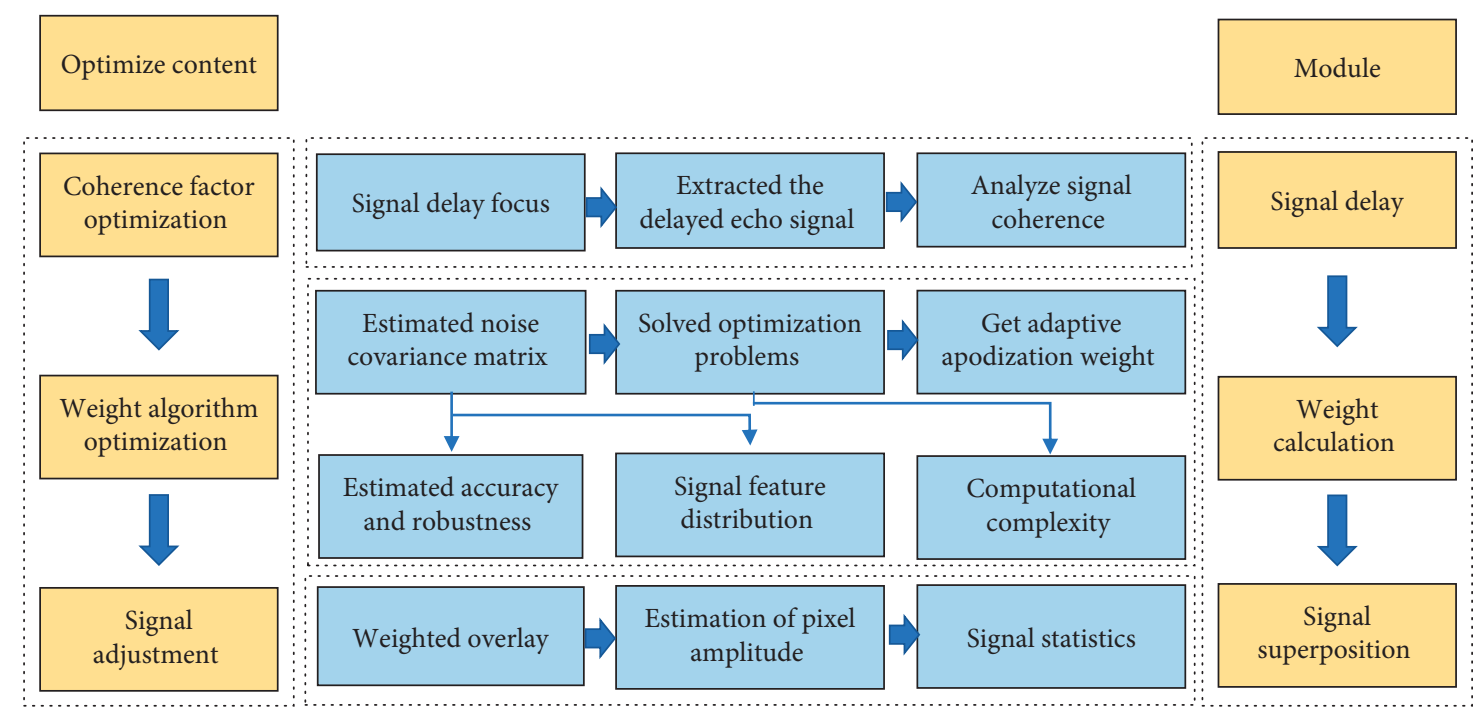

FIGURE 1: The basic flow and optimization of the adaptive ultrasound imaging algorithm.

$$
R=\frac{1}{2}\left(R_{f}+R_{b}\right) .
$$

The diagonal loading method can superimpose a certain proportion of the identity matrix on the noise covariance matrix. In the algorithm in (6), $\gamma$ is the diagonal loading, and the general value range is $\gamma \ll 1 / I$, which is taken as $\gamma \in[1 / 10 I+1 / 100 I]$ in this article; $K$ is the identity matrix; and trance is the trace of the matrix.

$$
R_{d}=R_{m}+\gamma \cdot \operatorname{trance}\left[R_{m}\right] \cdot K .
$$

The coherence factor is fused with the beamformer to suppress the side lobes and grating lobes [10]. In the algorithm of (7), $q$ adjusts the sensitivity of the coherence factor, while $p_{j}$ is the value of the sampling point.

$$
A_{s}=\left|1-\sqrt{1-\left(\frac{1}{N} \sum_{j=0}^{N-1} p_{j}\right)^{2}}\right|^{q} .
$$

2.4. Risk Factor Analysis Method of PICC-RT in Tumor Patients. The literature search method was adopted to analyze the risk factors of PICC-RT in tumor patients, and the search ended on December 1, 2019. It included China Journal Full-Text Database, WanFang Database, Chinese Biomedical Literature Database, PubMed, EM Base, and the Cochrane Library. Search terms were peripherally inserted central catheter (PICC), thrombosis, thromboembolism, etc. Inclusion criteria: I, age no less than 18 years; II, research on risk factors or predictors of PICC-RT in tumor patients; III, PICC-RT being diagnosed by ultrasound. Exclusion criteria: I, repeated publications, animal studies, and literature reviews; II, single-factor or multifactor regression analysis not conducted; III, studies that did not specify the number of subjects or did not obtain the $95 \%$ confidence interval and odds ratio.
2.5. Nursing Intervention Methods and Observation Indexes of Complicated PICC-RT. A PICC-equipped professional grip ball was distributed to all patients before taking PICC, and so did the upper extremity exercise education plan. One set of upper extremity exercise diary cards was adopted to record in detail the number of upper extremity exercises, arm swelling during exercise, and the degree of pain. The control group held the ball about 500 times a day: three times in the morning, midnight, and evening. Strength was the maximum grip strength that the patient could bear. At the same time, the patient was instructed to come to the hospital for an ultrasound examination for thrombosis immediately when he found pain in the arm on the side of the catheter, local fever, redness, and swelling during exercise. The observation group started to exercise 1 day before the catheterization and carried out ball holding exercises under the guidance of medical staff after three meals a day: extend the arm and the limb at an angle of $30^{\circ}-60^{\circ}$ and hold the grip ball with the tube side hand. When the grip ball was squeezed to $1 / 2$ of its original size, the patients should hold it firmly for 10 seconds and then release it for 10 seconds. Hold and loosen would be repeated 60 times a day, and patients should exercise every day until the catheter was removed. All subjects underwent venous Doppler color ultrasound examination on the limbs of the catheterized side before catheter placement, $1 \mathrm{~d}, 3 \mathrm{~d}, 7 \mathrm{~d}, 14 \mathrm{~d}, 21 \mathrm{~d}, 28 \mathrm{~d}$ after catheterization, and $1 \mathrm{~h}$ after exercise. The patient's thrombosis, thrombosis formation time, and complications were recorded.

2.6. Statistical Methods. The experimental data were processed using SPSS19.0 statistical software. The measurement data were expressed as mean plus or minus standard deviation $(\bar{x} \pm s)$, which was tested by $t$ test. Count data were expressed in percentage (\%). The $\chi^{2}$ test was used. $P<0.05$ indicated that the difference was statistically significant. 


\section{Results}

3.1. Image Quality Analysis Based on Adaptive Ultrasound Imaging Algorithm. The transverse resolutions of proposed ultrasonic imaging algorithm $(\mathrm{MV}-\mathrm{N})$, forward and backward smoothing operator (MV-FB), diagonal loading operator (MV-DL), eigenspace projection operator (MV-ESP), symbolic coherence coefficient operator (MV-SCF), and generalized coherence coefficient (MV-GCF) were compared (Figure 2). As the ratio of the length of the sliding window/number of elements (L/M) increased, the lateral resolution of the MV-N was in a stable state. MV and MVFB fluctuated greatly with the increase of $\mathrm{L} / \mathrm{M}$ value. The imaging contrast under these seven algorithms was analyzed (Figure 3), and the results showed that MV-N and MV-GCF were less affected by the $\mathrm{L} / \mathrm{M}$ value.

3.2. Analysis of Imaging Speed Based on Adaptive Ultrasound Imaging Algorithm. For the imaging calculation frame rate analysis under the seven algorithms (Figure 4), the MV-ESB algorithm was in a stable state with the increase of the L/M value. For the speedup results of GPU/CPU implementation of various algorithms (Figure 5), all algorithms had achieved speedups of more than a thousand times, and the speedup of the MV-N algorithm in this article was higher than other algorithms.

3.3. Analysis of Risk Factors of PICC-RT in Tumor Patients. According to the literature search, the risk factors of PICCRT were counted. In Table 1, the five aspects including basic characteristics, medical history, catheterization factors, biochemical indicators, and treatment factors of the patients were independent risk factors for PICC-RT, which were all statistically notable.

3.4. Tumor Patients Complicated by PICC-RT Imaging Examination. Ultrasound examinations were performed on all subjects before and after nursing intervention. The internal jugular vein could be clearly observed by ultrasound examination (shown in green in Figure 6(a)). The blood flow of the patient's probe movement was detected (Figures 6(b) and $6(\mathrm{c})$ ), and the patient's arterial and venous blood flow signals could be displayed (Figures 6(d) and 6(e)). The PICC and puncture needle images could be observed (Figures 6(f) and 6(g)), and PICC-RT (red arrow in Figure 6(h)) and the position of the catheter (open arrow in Figure 6(h)) could be displayed.

3.5. Contrast of Basic Data of Two Groups of Patients. In Table 2, the basic data of the two groups of patients were compared and analyzed. There was no obvious difference between the basic data of the two groups of patients $(P>0.05)$.

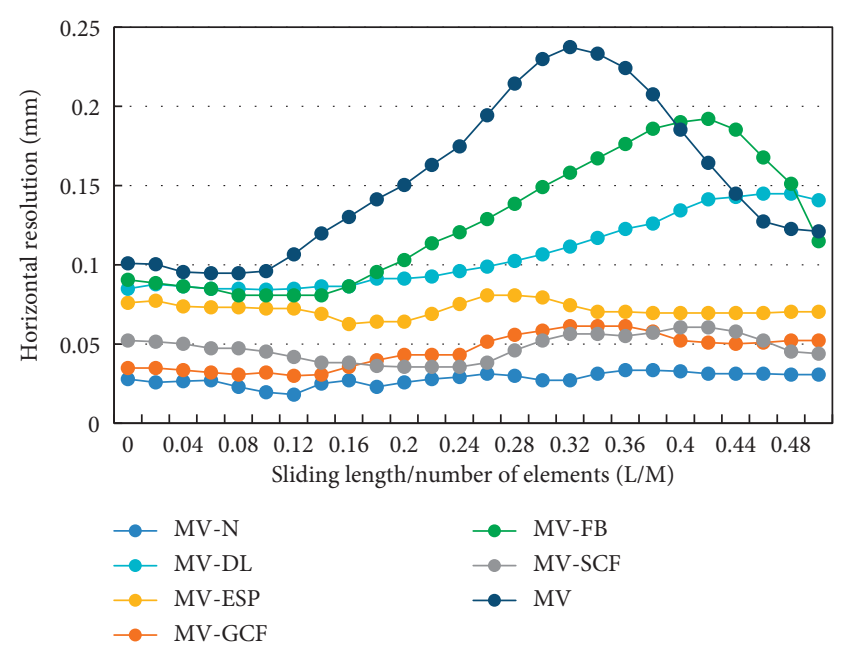

Figure 2: Contrast of ultrasound imaging resolution of different algorithms.

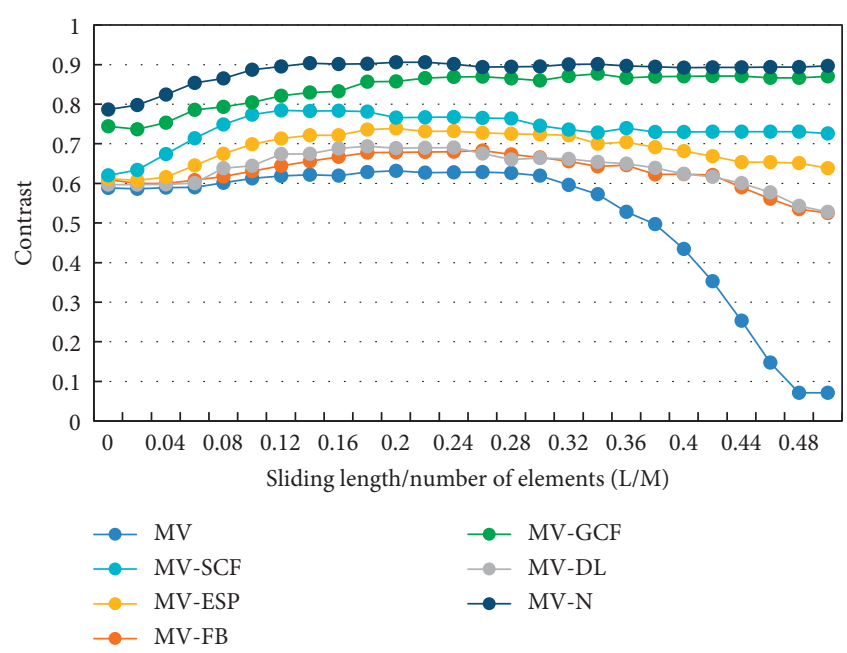

FIGURE 3: Comparison of ultrasound imaging contrast of different algorithms.

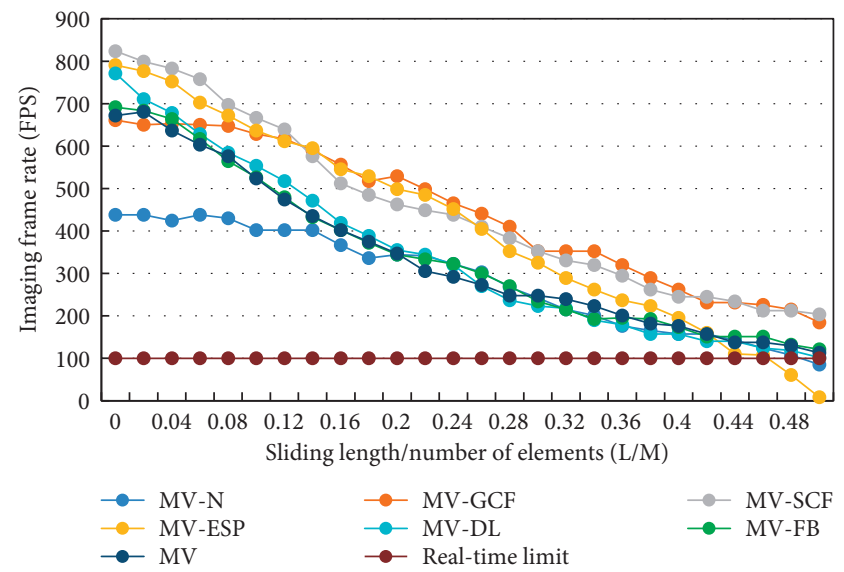

Figure 4: Contrast of the calculated frame rate of ultrasound imaging with different algorithms. 


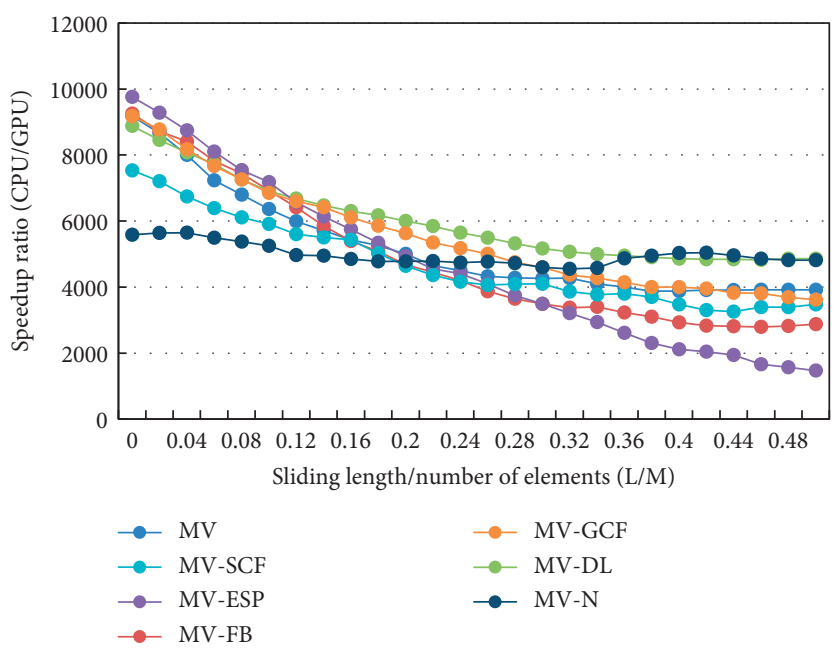

FIGURE 5: Analysis of speedup ratio of ultrasound imaging with different algorithms.

TABLE 1: PICC-RT risk factors analysis.

\begin{tabular}{|c|c|c|c|c|c|}
\hline Risk factor & $\chi^{2}$ & $I^{2} \%$ & OR & $95 \% \mathrm{CI}$ & $P$ \\
\hline Gender (male) & 0.06 & 0 & 2.17 & $(1.27,2.98)$ & $\leq 0.001^{*}$ \\
\hline Pathological type (lung cancer) & 3.67 & 19 & 3.25 & $(2.28,6.98)$ & $\leq 0.001^{*}$ \\
\hline Clinical stage (phase III-IV) & 7.26 & 54 & 4.17 & $(1.67,10.04)$ & $0.003^{*}$ \\
\hline Obesity $(\mathrm{BMI}>25)$ & 5.28 & 38 & 4.43 & $(2.65,6.76)$ & $\leq 0.001^{*}$ \\
\hline History of thrombosis & 4.65 & 69 & 2.68 & $(1.78,4.38)$ & $\leq 0.001^{*}$ \\
\hline Diabetes & 0.62 & 0 & 2.83 & $(1.72,4.62)$ & $\leq 0.001^{*}$ \\
\hline Intubation history & 4.53 & 23 & 2.47 & $(1.65,3.49)$ & $\leq 0.001^{*}$ \\
\hline History of chemotherapy & 7.72 & 49 & 1.59 & $(1.19,1.93)$ & $\leq 0.001^{*}$ \\
\hline Catheter type & 0.86 & 0 & 4.05 & $(1.46,7.65)$ & $\leq 0.001^{*}$ \\
\hline Tube placement (cephalic vein) & 32.07 & 87 & 3.61 & $(1.53,674)$ & $0.001^{*}$ \\
\hline Catheter tip position & 4.25 & 23 & 4.97 & $(3.09,8.19)$ & $\leq 0.001^{*}$ \\
\hline FIB $(>4 \mathrm{~g} / \mathrm{L})$ & 12.07 & 73 & 1.69 & $(0.67,3.98)$ & $\leq 0.001^{*}$ \\
\hline Use of anticoagulant drugs & 42.66 & 87 & 3.09 & $(1.29,3.86)$ & $0.005^{*}$ \\
\hline D-dimer $(\mathrm{D}-\mathrm{D})(>0.5 \mathrm{mg} / \mathrm{L})$ & 13.28 & 52 & 2.62 & $(2.02,3.82)$ & $\leq 0.001^{*}$ \\
\hline
\end{tabular}

*The difference was statistically notable.

3.6. Statistics of the Exercise of the Two Groups of Patients. The exercise status of the two groups of patients is shown in Figure 7 . The exercise completion rate of the observation group was $100 \%$ within 28 days, and the completion rate of the control group began to decrease from day 14 and dropped to $82.08 \%$ at day 28 .

3.7. Contrast of Average Flow Velocity per Unit Time of Axillary Vein between Two Groups. In Figure 8, the observation group had no notable difference in the average flow velocity per unit time of the axillary vein of patients within 28 days and that before intubation (0d). The average flow velocity per unit time in the axillary vein of the control group continued to decrease, and there was notable difference between that at day 28 and before the intubation, $P<0.05$.

3.8. Contrast of Occurrence of PICC-RT between the Two Groups. The results of real-time monitoring of PICC-RT after catheterization in the two groups are shown in Table 3 and Figure 9. The total number of cases and the KaplanMeier curve were notably different $(P<0.05)$. The occurrence of PICC-RT complications in the two groups was shown in Table 3. There was remarkable difference between the two groups $(P<0.05)$. The incidence of PICC complications in the control group was obviously higher in contrast to observation group $(P<0.05)$.

\section{Discussion}

Based on MV algorithm, smoothing technique algorithm, diagonal loading algorithm, and correlator algorithm were optimized. Then the image obtained by the optimized algorithm was compared with other algorithms. It was found that the image lateral resolution and imaging contrast obtained by the MV-N algorithm were relatively stable under different $\mathrm{L} / \mathrm{M}$ values. The results showed that the robustness of the MV algorithm was improved and the imaging stability was increased. MV-N algorithm's GPU imaging calculation frame rate was more stable, and the acceleration was higher than other algorithms. It meant that MV-N could improve image quality and realize real-time imaging.

Moreover, there was no difference in the probability of first occurrence of venous thrombosis between different 

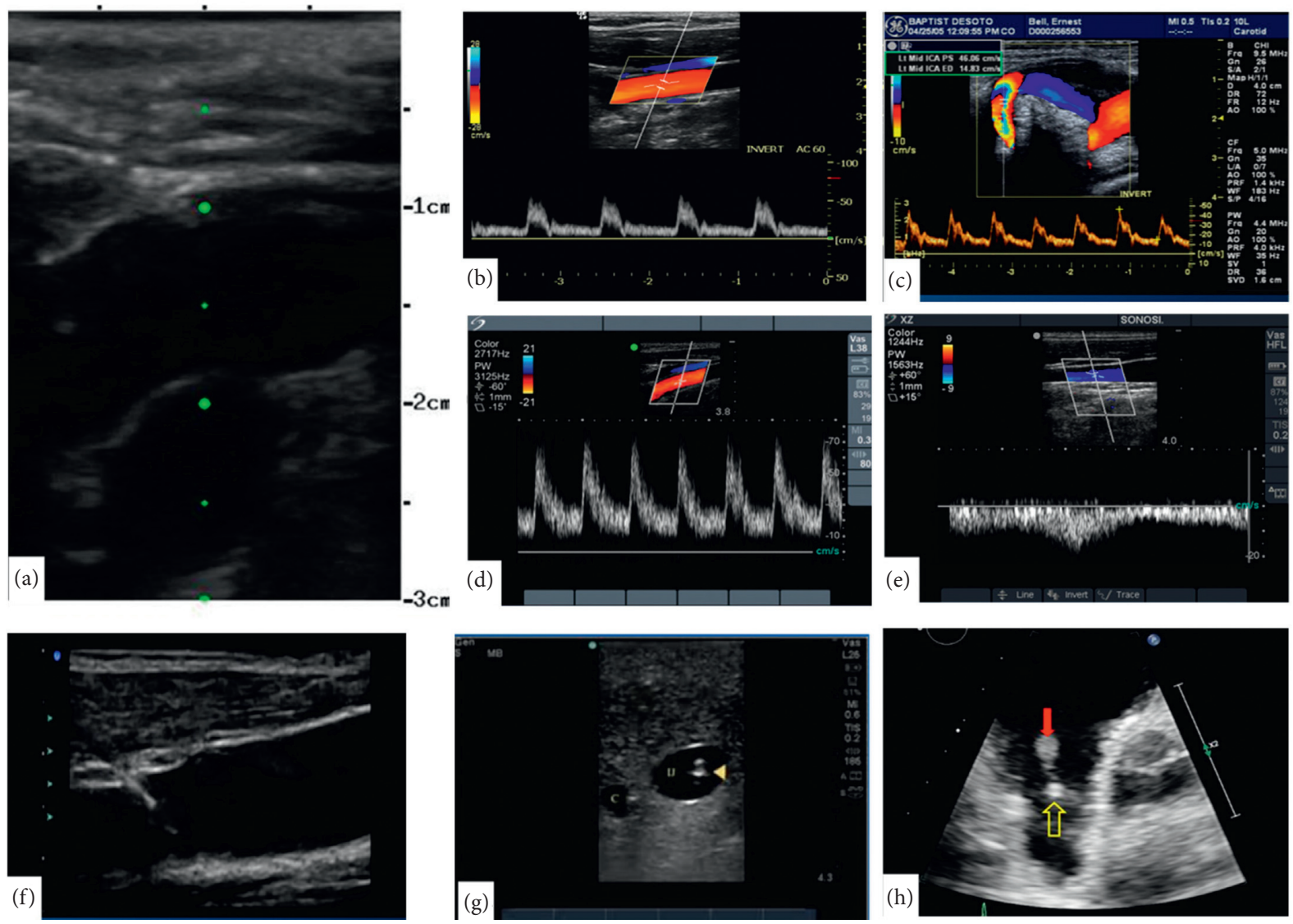

FIgURE 6: Ultrasound examination of PICC-RT in tumor patients. (a) Ultrasound image of internal jugular vein; (b) blood flow running in the direction of the probe; (c) blood flow moving away from the direction of the probe; (d) pulsed blood flow signal; (e) venous blood flow signal; (f) PICC image; (G) puncture needle image; (h) PICC terminal thrombosis.

TABle 2: Contrast of basic data of the two groups of patients.

\begin{tabular}{|c|c|c|c|c|}
\hline Item & Control group & Observation group & $T / \chi^{2}$ & $P$ \\
\hline$\overline{\text { Age }}$ & $54.28 \pm 6.23$ & $55.19 \pm 5.93$ & 0.287 & 0.563 \\
\hline Gender (female) & $33(37.08 \%)$ & $36(39.56 \%)$ & 0.419 & 0.525 \\
\hline BMI & $23.17 \pm 2.72$ & $24.18 \pm 2.65$ & -0.832 & 0.884 \\
\hline Health score & $84.19 \pm 9.98$ & $85.52 \pm 10.13$ & -0.793 & 0.402 \\
\hline Breast cancer & $27(30.34 \%)$ & $29(31.87 \%)$ & 0.529 & 0.418 \\
\hline Gastrointestinal cancer & $36(40.45 \%)$ & $34(37.36 \%)$ & 0.515 & 0.411 \\
\hline Respiratory tract tumor & $19(21.35 \%)$ & $22(24.18 \%)$ & 0.503 & 0.405 \\
\hline Others & $7(7.87 \%)$ & $6(6.59 \%)$ & 0.496 & 0.392 \\
\hline $0-6$ months & $49(55.06 \%)$ & $52(57.14 \%)$ & 0.023 & 0.905 \\
\hline More than 6 months & $40(44.94 \%)$ & $39(42.86 \%)$ & 0.018 & 0.925 \\
\hline Pulmonary vein & $31(34.83 \%)$ & $33(36.26 \%)$ & 1.323 & 0.942 \\
\hline Median cubital vein & $22(24.72 \%)$ & $19(20.88 \%)$ & 1.275 & 0.918 \\
\hline Brachial vein & $36(40.45 \%)$ & $39(42.86 \%)$ & 1.292 & 0.926 \\
\hline Catheter in left arm & $47(52.81 \%)$ & $50(54.95 \%)$ & 0.117 & 0.542 \\
\hline Catheter in right arm & $42(47.19 \%)$ & $41(45.05 \%)$ & 0.121 & 0.498 \\
\hline Catheter length & $44.25 \pm 5.29$ & $43.62 \pm 5.92$ & -0.498 & 0.623 \\
\hline Arm circumference & $27.18 \pm 2.21$ & $25.97 \pm 2.65$ & 0.291 & 0.765 \\
\hline
\end{tabular}

genders, and women aged 20-35 had a higher probability of occurrence of venous thrombosis [11, 12]. Stewart et al. [13] pointed out that there were differences in the responses of different genders to anticoagulant therapy. Hisada et al. [14] suggested that obese patients were more likely to have abnormal coagulation factors, so the probability of venous thrombosis was higher. This statistical analysis revealed that BMI higher than 25 was a risk factor for PICC-RT in tumor patients. It may be related to the fact that obese patients had less exercise and had blood viscosity and blood stasis [15], which increased the risk of blood clots. Leeds et al. [16] and Horner et al. [17] pointed out that patients with 


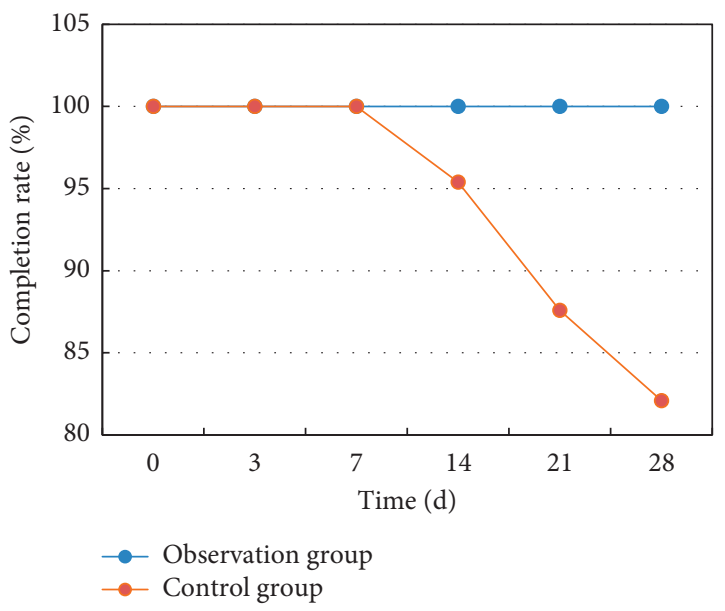

Figure 7: Analysis of the exercise of the two groups of patients.

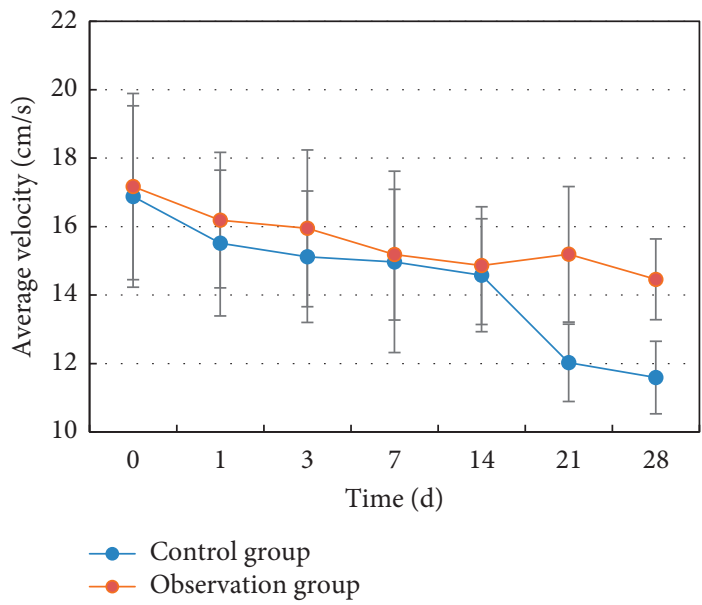

Figure 8: Contrast of the average flow velocity of the axillary vein per unit time between the two groups.

TABLE 3: Contrast of occurrence of PICC-RT between the two groups (cases).

\begin{tabular}{lccccccccc}
\hline & $1 \mathrm{~d}$ & $3 \mathrm{~d}$ & $7 \mathrm{~d}$ & $14 \mathrm{~d}$ & $21 \mathrm{~d}$ & $28 \mathrm{~d}$ & Sum & $\chi^{2}$ & $P$ \\
\hline Control group & 0 & 2 & 1 & 3 & 5 & 4 & 15 & 6.023 & 0.014 \\
Observation group & 0 & 2 & 0 & 0 & 0 & 0 & 2 & & \\
$P$ & & 1.00 & 0.51 & 0.12 & 0.03 & 0.08 & 0.013 & & \\
\hline
\end{tabular}

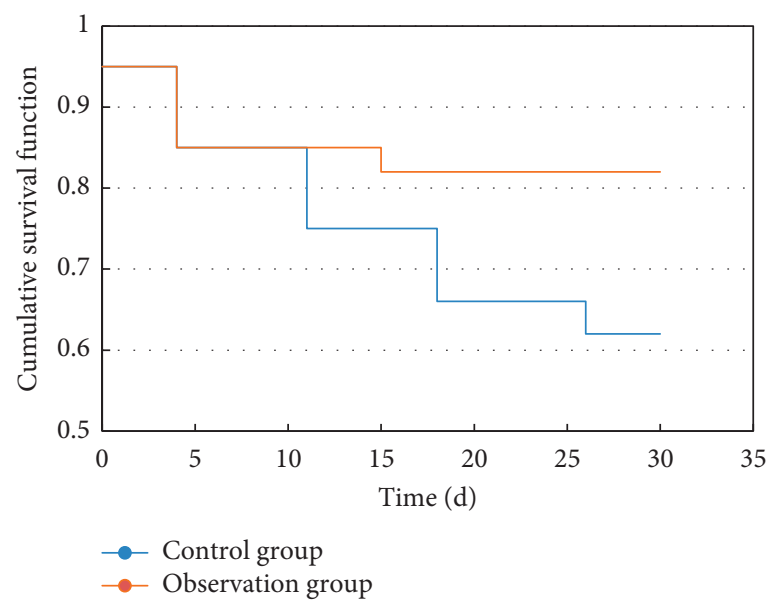

Figure 9: Kaplan-Meier curve of two groups of patients 
adenocarcinoma had a higher risk of venous thrombosis. This study found that adenocarcinoma was an independent risk factor for PICC-RT. It may be because that the increased mucin level of adenocarcinoma cells leaded to abnormal expression of the coagulation system, resulting in thrombosis [18]. The exercise regimens of patients with tumor PICC were refined, and the probability of PICC-RT occurrence was compared between the observation group and the control group. The results showed that the incidence of PICC-RT and its complications in the observation group was notably lower, which was similar to the results of $\mathrm{Yu}$ et al. [19].

\section{Conclusion}

In this work, MV algorithm was utilized to optimize the ultrasonic image quality and image processing speed, and the risk factors for patients with tumor complicated with PICC-related thrombosis were discussed, so did the intervention effect of upper limb movement method. However, there are still some deficiencies in this research. There was no statistical analysis of the thrombosis sites of the study subjects. In the later study, the relevant thrombosis sites of the study subjects will be further statistically analyzed. To sum up, a novel algorithm that could increase the ultrasonic image quality and calculation speed was established. Based on the risk factors for PICC-RT, a kind of upper limb movement that could reduce the occurrence of PICC-RT and its complications was proposed.

\section{Data Availability}

No data were used to support this study.

\section{Conflicts of Interest}

The authors declare no conflicts of interest.

\section{References}

[1] J. Jaffray, C. Witmer, S. H. O'Brien et al., "Peripherally inserted central catheters lead to a high risk of venous thromboembolism in children," Blood, vol. 135, no. 3, pp. 220-226, 2020.

[2] P. Balsorano, G. Virgili, G. Villa et al., "Peripherally inserted central catheter-related thrombosis rate in modern vascular access era-when insertion technique matters: a systematic review and meta-analysis," The Journal of Vascular Access, vol. 21, no. 1, pp. 45-54, 2020.

[3] N. J. Brandmeir, J. R. Davanzo, R. Payne et al., "A randomized trial of complications of peripherally and centrally inserted central lines in the neuro-intensive care unit: results of the NSPVC trial," Neurocritical Care, vol. 32, no. 2, pp. 400-406, 2020.

[4] T. M. Kleidon, C. M. Rickard, J. A. Schults et al., "Development of a paediatric central venous access device database: a retrospective cohort study of practice evolution and risk factors for device failure," Journal of Paediatrics and Child Health, vol. 56, no. 2, pp. 289-297, 2020.

[5] N. Scrivens, E. Sabri, C. Bredeson, and S. McDiarmid, "Comparison of complication rates and incidences associated with different peripherally inserted central catheters (PICC) in patients with hematological malignancies: a retrospective cohort study," Leukemia and Lymphoma, vol. 61, no. 1, pp. 156-164, 2020.

[6] N. Marsh, E. Larsen, S. Tapp, M. Sommerville, G. Mihala, and C. Rickard, "Management of hospital in the home (HITH) peripherally inserted central catheters: a retrospective cohort study," Home Health Care Management \& Practice, vol. 32, no. 1, pp. 1-6, 2020.

[7] M. D. Weber, B. Eithun, A. S. Himebauch, and T. Conlon, "Cephalic peripherally inserted central catheter placement with retrograde basilic vein malposition," The Journal of Vascular Access, vol. 21, no. 1, pp. 125-126, 2020.

[8] K.-V. Chang, W.-T. Wu, and L. Özçakar, "Ultrasound imaging for a rare cause of postpartum forearm pain: diffuse enlargement rather than focal swelling of the deep branch of the radial nerve," Pain Medicine, vol. 21, no. 1, pp. 203-205, 2020.

[9] H. J. Park, H. Y. Jang, S. Y. Kim et al., "Non-enhanced magnetic resonance imaging as a surveillance tool for hepatocellular carcinoma: comparison with ultrasound," Journal of Hepatology, vol. 72, no. 4, pp. 718-724, 2020.

[10] S. Harput, K. Christensen-Jeffries, A. Ramalli et al., "3-D Super-resolution ultrasound imaging with a 2-D sparse array," IEEE Transactions on Ultrasonics, Ferroelectrics, and Frequency Control, vol. 67, no. 2, pp. 269-277, 2020.

[11] J. Greffier, J. Frandon, F. Pereira et al., "Optimization of radiation dose for $\mathrm{CT}$ detection of lytic and sclerotic bone lesions: a phantom study," European Radiology, vol. 30, no. 2, pp. 1075-1078, 2020.

[12] S. Konstantinides, S. Konstantinides, G. Meyer et al., "ESC Guidelines for the diagnosis and management of acute pulmonary embolism developed in collaboration with the European respiratory society (ERS)," European Heart Journal 2020, vol. 41, no. 4, pp. 543-603, 2019.

[13] L. K. Stewart and J. A. Kline, "Metabolic syndrome increases risk of venous thromboembolism recurrence after acute deep vein thrombosis," Blood Advances, vol. 4, no. 1, pp. 127-135, 2020.

[14] Y. Hisada, S. P. Grover, A. Maqsood et al., "Neutrophils and neutrophil extracellular traps enhance venous thrombosis in mice bearing human pancreatic tumors," Haematologica, vol. 105, no. 1, pp. 218-225, 2020.

[15] R. Chiari, B. Ricciuti, L. Landi et al., "ROS1-rearranged nonsmall-cell lung cancer is associated with a high rate of venous thromboembolism: analysis from a phase II, prospective, multicenter, two-arms trial (METROS)," Clinical Lung Cancer, vol. 21, no. 1, pp. 15-20, 2020.

[16] I. L. Leeds, J. K. Canner, S. R. Dibrito, and B. Safar, "Justifying total costs of extended venothromboembolism prophylaxis after colorectal cancer surgery," Journal of Gastrointestinal Surgery, vol. 24, no. 3, pp. 677-687, 2020.

[17] D. Horner, J. W. Stevens, A. Pandor et al., "Pharmacological thromboprophylaxis to prevent venous thromboembolism in patients with temporary lower limb immobilization after injury: systematic review and network meta-analysis," Journal of Thrombosis and Haemostasis, vol. 18, no. 2, pp. 422-438, 2020.

[18] Y. Zhang, Z. Zhang, R. Wei et al., "IL (Interleukin)-6 Contributes to deep vein thrombosis and is negatively regulated by miR-338-5p," Arteriosclerosis, Thrombosis, and Vascular Biology, vol. 40, no. 2, pp. 323-334, 2020.

[19] H. B. Yu, A. Bartlett, P. Hunter, and H. Ho, "Computational simulations for the hepatic arterial buffer response after liver graft transplantation from an adult to a child," Medical Engineering \& Physics, vol. 75, pp. 49-52, 2020. 